\title{
Research and Survey on Applications of Wireless Techniques for Electrical Monitoring and Control Systems
}

\author{
LIN Jin ${ }^{1, a}$ \\ ${ }^{1}$ Institute of mechanical and electrical technology, Wuxi institute of commerce, \\ Wuxi 214153,China \\ alinjinwx@126.com
}

Keywords: Wireless and Communication Technology; Electrical Monitoring; Control System; Zigbee Technique; Smart Grid; IEEE Standards.

\begin{abstract}
In this paper, we finalize the research on the applications of wireless techniques for electrical monitoring and control systems. Monitoring system plays an important role in our daily life. Load frequency control method is widely used in the network can be complex, distributed generation in a large geographic area. Using frequency control can share power without any communication between distributed generators. Real and reactive power sharing can control the amount of two independent basic frequency and voltage level. Some research institutions at home and abroad have begun to wireless sensor network applications in the field of aquaculture. We design and implement the novel system with the Zigbee technique and the related internet of the things (IoT) technique. The experimental result indicates that our system outperforms other systems. The covering area and the accuracy is beyond the average standard.
\end{abstract}

\section{Introduction}

Improving power flow management in distributed generation is essential for high system reliability and accurate control of power sources. Monitoring system plays an important role in our daily life. [1-5] Load frequency control method is widely used in the network can be complex, distributed generation in a large geographic area. Using frequency control can share power without any communication between distributed generators. Real and reactive power sharing can control the amount of two independent basic frequency and voltage level. Some research institutions at home and abroad have begun to wireless sensor network applications in the field of aquaculture, including: the application of multi-parameter water quality sensor and ZigBee wireless sensor network technology to create the aquaculture water quality test and control integration system; The ZigBee technology combined with GPRS communication [6]; The bus technology and GPRS communication technology; Wireless sensor network applied in aquaculture water quality monitoring. In order to further improve the reliability of the remote online monitoring system of aquaculture, anti-jamming and flexibility, this paper designs a wireless multi-parameter monitoring system based on ZigBee protocol. The system adopts the Chipcon company produces the wireless transceiver chip CC2430 and build the wireless network using the wireless communication technology, without setting any lines, automatic networking, low cost, collection of data can be pictures by monitoring the real-time monitoring, effective implementation of the various parameters of water detection. To improve system performance and overcome the challenges in power management, communication among the DGs and control centers is essential.

ZigBee technology is a low rate, at close range, low power consumption, low complexity and low cost, reliable communication and network characteristics of large capacity of the line connected to the wireless network communication technology and control of protocol specification, a set of very complete hierarchy, formulated jointly by the IEEE 802 and ZigBee alliance. It works to avoid paying and avoid application of $2.4 \mathrm{GHz}$ ISM band, has a long battery life, simple application and high reliability, networking ability, etc. Based on the ZigBee wireless sensor network (WSN) usually consists of the coordinator, routers and terminal equipment of three kinds of node equipment. The 
monitoring network consists of several coordinator nodes, number of sensor nodes, a number of control nodes and a monitoring center. Within range of the coordinator node and sensor node USES the lowest energy consumption of the star network topology. There is only one node in the network as a coordinator, responsible for the organization and maintenance of network, other nodes can be used as a router and terminal equipment. Router is responsible for routing, network information terminal equipment is realizing the function of sensor node, the coordinator and the router also has the function of allow devices to join or leave the network. Information about the power delivery of the sources in a power system with high penetration level of distributed generators (DGs) based on renewable energy resources, is becoming essential for planning and allocating resources. ZigBee network support: three kinds of topology star network (star), the tree network (tree) and a mesh network (mesh), can choose according to the practical application of different network topology [7]. In this paper, we will conduct research on applications of wireless techniques for electrical monitoring and control systems. The detailed description of our methodology will be introduced in the following sections.

\section{Our Proposed Techniques}

The General Structure of the Designed System. For remote power control and monitoring, many wireless technologies are discussed; these are: Infrared rays, WLAN, Bluetooth, and Zigbee. General electrical appliances have three power modes, the normal, just stand by. In normal mode, the household appliances using maximum power, however, in the standing in the model, the household appliances use nearly $10 \%$ of the energy consumption than the normal mode [8].

The coordinator is finalized by RS.232 serial port which is connected to a PC. Microsoft Visual Basic 6.0 as development tool, is used in the development of a PC communication program, it provides a variety of ways to realize serial communication. System uses Windows provide control to develop serial communication program, its operation is simple, powerful. Using the properties of the controls for a serial port Settings, event driven to a serial port response of the control, the control method to complete the serial port can send and receive data. The author uses the VB language monitor screens to help real-time monitoring to collect data, realizes the visual man-machine interface, convenient debugging and network monitoring system. In addition, the system choose SQL server 2000 as the database, save every time acquisition of data, convenient operators call history data for analysis. Embedded board is used instead of computer to reduce power consumption. In case of overload/abnormality in power consumption, server module sends a control message to MCU via Zigbee module. MCU cuts power to the load for safety purpose. The figure 1 shows the structure.

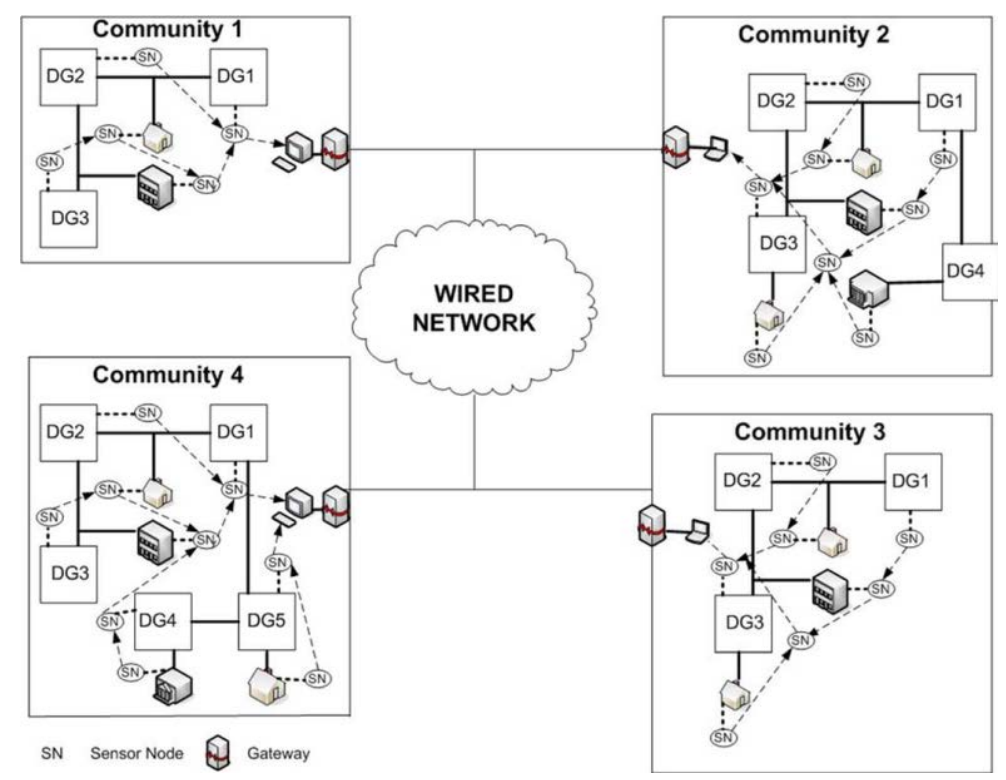

Fig.1 The Structure of the Proposed System 
The Hardware Description. All the DGs are interfaced though voltage source converter. The converters are implemented with three $\mathrm{H}$ Bridges of IGBT switches and controlled with PWM modulation. The $\mathrm{H}$ bridges are connected as shown in Fig. 2. Note that even half bridge connection will serve for the purpose. But in many cases, a full bridge structure is preferred, because it can be controlled separately in each phase is very effective compensation. In practical applications, the sensor node, the coordinator and PC software design is the most important. Data collection using the I2C protocol, sensor node USES the ZigBee wireless communication protocol between the sensor nodes to collect data sent to the coordinator. In addition, we through the use of Visual Basic programming environment provides version 6.0 control pattern to realize the upper machine and lower machine communication. The transformer can provide much-needed isolation and voltage increase. Verify the routing protocol is proposed four scenarios. The first case displays the packet success rate and the end-to-end delay when the AODV routing protocol. The second case shows that packet success rate and the end-to-end delay when OSLR as routing protocols. The third case shows the end-to-end delay and packet success than AODV in the middle of the target node failure. The fourth case is packet success rate and the end-to-end delay in the target node FRL failure of an intermediate node.

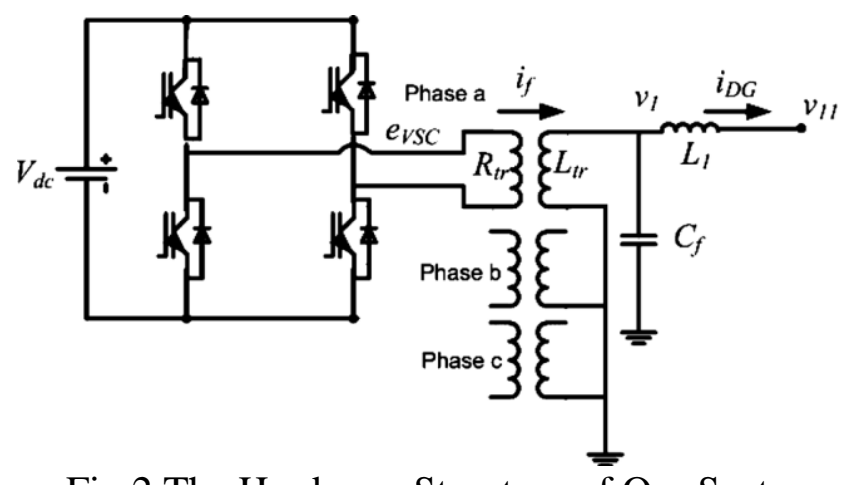

Fig.2 The Hardware Structure of Our System

The Transmission Section. Zigbee technology can be implemented in generation, transmission, distribution, and in consumer sectors to provide control on power consumption and give accurate data to user and utility. Zigbee based devices have two types: one is Full Function Device (FFD) and other is RFD. FFD can be coordinator or router. It is liable to create the network, select the radio frequency channel and distinctive network identifier to avoid collision among data from different sensor nodes and for security purpose. Zigbee RFD plays role of end device that collects variety of data from different switches and sensors. Here we discuss Zigbee based transmission lines monitoring, meter reading system and load control system. Tower of transmission line monitoring system is based on lines and environment parameters such as temperature, ice, wind, lightning sensors placed in transmission lines. These based on Zigbee sensor power through it and send to the data center through the Zigbee is fixed in the transmission tower gateway. It provides two small communities located far apart. Wireless network has two, one in each community, they through cable network connection. The director-general and load power in a community CC1 control center by wireless communication network and CC2. The international community data exchange using wired network shown in the diagram. Coordinator in electricity will have initialization state, trigger the key events to determine equipment as the coordinator, start a ZigBee network, network monitoring and then enter the wait state. When, after receipt of the child nodes access request for its distribution network address, and send children equipment hook confirmation message, establish a binding. This saves the time that is required to set a new routing path in order to route the packet to the destination. The static route can be predetermined so that the least number of hops is required between the source and the destination. Subsequently, the coordinator for data request, when received after sending the data information of sensor nodes, analyze the data packets, after confirm the information for the data, the data to the liquid crystal display (LCD) and PC interface display. User sends control command message through GSM cellular mobile phone to PMS. After receiving control command PMS allows user to control if sending command format match with system. The figure 3 describes the process. 


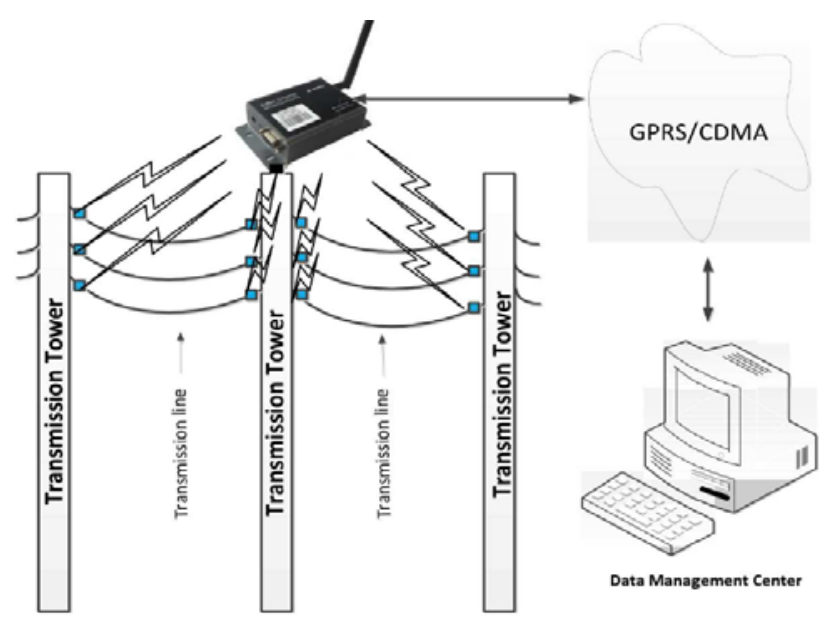

Fig.3 The Transmission Pattern of the System

\section{Simulation Result and Experiment}

This section examines the effect of increase in number of connections and number of hops on the important parameters such as end-to-end delay and number of packets received. The proposed application of WSN in a micro-grid requires high packet success ratio and low end-to-end delay. Simulation results indicate that reactive routing protocols such as AODV have a significant end-to-end delay [9-10], which increases further with failing nodes. FRL reduces packet loss by switching to static and a default route if the next hop forwarding node fails. This saves the time spent on determining a new routing path as in AODV. Since this application does not consider mobile nodes configuring the nodes with static and default routes is a feasible solution. The figure 5 shows the test. User can be capable for supervise and organize power condition of electric home appliances anytime and anywhere using GSM cellular mobile. We could conclude from the figure 5 that our proposed system pattern will cover most of the areas.

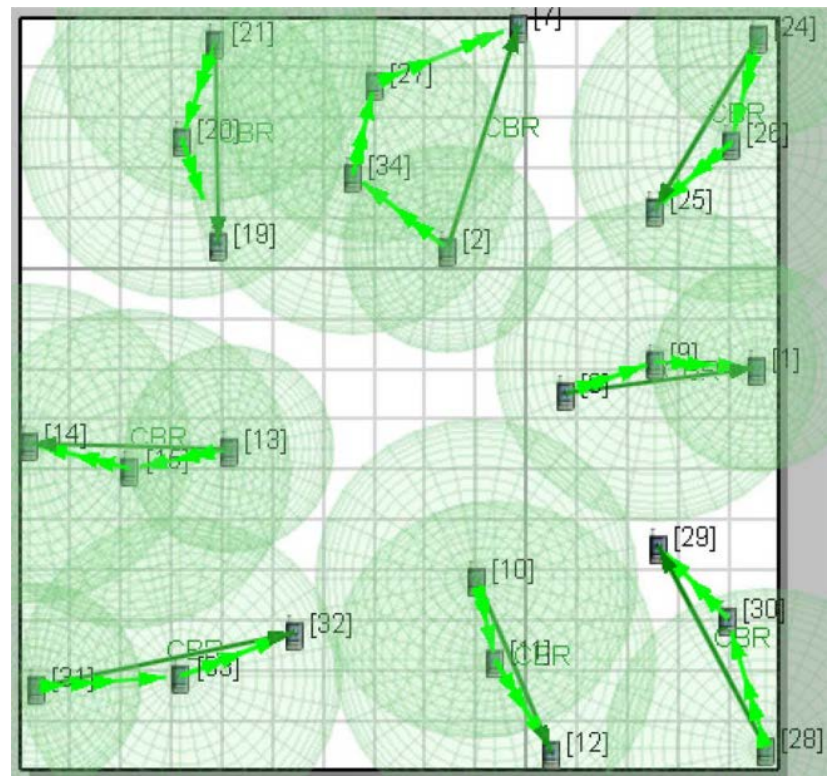

Fig.5The Comparison Result of the Technique

\section{Conclusion and Summary}

Improving power flow management in distributed generation is essential for high system reliability and accurate control of power sources. ZigBee technology is a low rate, at close range, low power consumption, low complexity and low cost, reliable communication and network characteristics of large capacity of the line connected to the wireless network communication 
technology and control of protocol specification, a set of very complete hierarchy, formulated jointly by the IEEE 802 and ZigBee alliance. In this research paper, we finalize the research on the applications of wireless techniques for electrical monitoring and control systems. For remote power control and monitoring, many wireless technologies are discussed; these are: Infrared rays, WLAN, Bluetooth, and Zigbee. General electrical appliances have three power modes, the normal, just stand by. Our proposed system is well-performed compared with other popular methods. The experimental result proves the effectiveness of our system. In the future, we will set up schedule for optimizing the network structure to gain higher accuracy and efficiency.

\section{References}

[1] Stamatescu G, Stamatescu I, Arghira N, et al. Embedded networked monitoring and control for renewable energy storage systems[J]. Development and Application Systems, International Conference on, 2014:1 - 6 .

[2] Hemami H H M. Modelling and monitoring of passive control structures in human movement[J]. International Journal of Control, 2014, 87(9):1861-1876.

[3] Wang H, Shkjezi F, Hoxha E. Distance metric learning for multi-camera people matching[C]. //Advanced Computational Intelligence (ICACI), 2013 Sixth International Conference on. IEEE, 2013:140 - 143.

[4] Kuo C, Sun S, Chang P. A skeleton-based pairwise curve matching scheme for people tracking in a multi-camera environment[J]. Signal and Information Processing Association Annual Summit and Conference (APSIPA), 2013 Asia-Pacific, 2013:1 - 5.

[5] Sepulchro W D N, Encarnação L F, Brunoro M. Harmonic State and Power Flow Estimation in Distribution Systems Using Evolutionary Strategy[J]. Journal of Control, Automation and Electrical Systems, 2014, 25(3):358-367.

[6] Jiang J, Wang Z, Ma H, et al. Remote Monitoring and Control System for Electrical Haulage Shearer[J]. Manufacturing and Applied Research, 2014.

[7] Silva T M P, Junior C D M. Self-Powered Active Control for an Aeroelastic Plate-Like Wing Using Piezoelectric Material[C]. //Modeling, Simulation and Control of Adaptive Systems. 2014:V001T03A018.

[8] K. Dash P, K. Barik S, K. Patnaik R. Detection and Classification of Islanding and Nonislanding Events in Distributed Generation Based on Fuzzy Decision Tree[J]. Journal of Control, Automation and Electrical Systems, 2014, 25(6):699-719.

[9] Morie K P, Sanctis P D, Foxe J J. Reward contingencies and the recalibration of task monitoring and reward systems: a high-density electrical mapping study.[J]. Neuroscience, 2014:100-117.

[10]Costa B S J, Angelov P P, Guedes L A. Real-Time Fault Detection Using Recursive Density Estimation[J]. Journal of Control, Automation and Electrical Systems, 2014, 25(4):428-437. 